\title{
Origin of the checkerboard pattern in scanning tunneling microscopy maps of underdoped cuprate superconductors
}

\author{
Kai-Yu Yang, ${ }^{1}$ Wei-Qiang Chen, ${ }^{2}$ T. M. Rice, ${ }^{1,3}$ and Fu-Chun Zhang ${ }^{2,3}$ \\ ${ }^{1}$ Institut für Theoretische Physik, ETH Zürich, CH-8093 Zürich, Switzerland \\ ${ }^{2}$ Center for Theoretical and Computational Physics and Department of Physics, The University of Hong Kong, Hong Kong, SAR, China \\ ${ }^{3}$ Kavli Institute for Theoretical Physics, University of California, Santa Barbara, California 93106, USA
}

(Received 4 August 2009; published 6 November 2009)

\begin{abstract}
The checkerboard pattern in the differential conductance maps on underdoped cuprates appears when the scanning tunneling microscopy is placed above the $\mathrm{O}$ sites in the outermost $\mathrm{CuO}_{2}$ plane. In this position the interference between tunneling paths through the apical ions above the neighboring $\mathrm{Cu}$ sites leads to an asymmetric weighting of final states in the two antinodal regions of $\boldsymbol{k}$ space. The form of the asymmetry in the differential conductance spectra in the checkerboard pattern favors asymmetry in the localization length rather than a nematic displacement as the underlying origin.
\end{abstract}

DOI: 10.1103/PhysRevB.80.174505

PACS number(s): 74.72.-h, 74.25.Jb, 74.62.Dh

\section{INTRODUCTION}

The large scale detailed maps of the tunneling density of states (DOS) in underdoped cuprates obtained recently by scanning tunneling microscopy (STM) have aroused great interest. ${ }^{1}$ This surface sensitive technique to date has been limited to strongly disordered BSCCO and $\mathrm{Ca}_{2-x} \mathrm{Na}_{x} \mathrm{CuO}_{2} \mathrm{Cl}_{2}$ samples. An analysis of these DOS maps found a local breaking of the square symmetry of the underlying lattice to form a checkerboard pattern. The presence of this checkerboard pattern in these two different underdoped cuprates has been interpreted as evidence for the presence of an intrinsic bond-centered electronic glass with unidirectional domains as a key characteristic of the pseudogap phase in underdoped cuprates. $^{2}$ A number of different interpretations of this pattern have also been suggested in the literature. ${ }^{3-9}$ In our study we re-examine possible origins for this pattern in light of very recent STM experiments. ${ }^{10}$

The new STM data taken in the superconducting state at low temperatures show that the checkerboard pattern appears most prominently at voltages where the tunneling processes are predominantly into antinodal regions in $\boldsymbol{k}$ space. At lower voltages the spatial pattern of the DOS maps is quite different. The low-energy pattern in the superconducting state was successfully analyzed as arising from interference generated by the scattering of propagating Bogoliubov quasiparticles in the presence of weak disorder. ${ }^{11}$ Kohsaka et al. ${ }^{10}$ found that a rapid change in the spatial pattern in the DOS maps occurred at the tunneling voltage corresponding to the energy of nodal centered arcs of Bogoliubov quasiparticles at their end points on the diamond formed by the lines in $\boldsymbol{k}$ space connecting the antinodal points. Thus the checkerboard pattern which appears at higher voltages is formed by the tunneling of electrons and holes into pseudogap state located in the antinodal regions of $\boldsymbol{k}$ space. The key question to be answered is the origin and interpretation of this checkerboard pattern.

The checkerboard pattern is characterized by a local symmetry breaking which reduces the square $C_{4}$ symmetry of the $\mathrm{Cu}-\mathrm{O}-\mathrm{Cu}$ bonds in a $(\mathrm{CuO})_{4}$ square plaquette to $C_{2}$ symmetry leading to a unidirectional pattern of domains with a glassy short-range order on the length scale of $4 a$ ( $a$ : lattice parameter). This short-range order with local symmetry breaking has led to proposals that a static spincharge stripe glass coexists with the superconductivity. $5,6,8,9$ Another set of proposals $3,4,7$ are based on the existence of static fluctuations in an order parameter which breaks both translational and $C_{4}$ symmetry leading to strong scattering of the Bogoliubov quasiparticles consistent with the observations. At this point we should comment on the difficulties associated with an explanation based on an intrinsic symmetry breaking. While it is true that the STM experiments were performed on the highly disordered cuprates, $\mathrm{BSCCO}$ and $\mathrm{Ca}_{2-x} \mathrm{Na}_{x} \mathrm{CuO}_{2} \mathrm{Cl}_{2}$, because of their good surfaces. Not all underdoped cuprates are disordered, for example, the two members of the YBCO family, $\mathrm{YBa}_{2} \mathrm{Cu}_{4} \mathrm{O}_{8}$ and $\mathrm{YBa}_{2} \mathrm{Cu}_{3} \mathrm{O}_{6.5}$-ortho-II, are well ordered and intrinsically strongly underdoped with hole concentrations estimated to be $x=0.14$ and 0.1 , respectively. Indeed samples of these cuprates are of sufficient quality to allow the observation of quantum oscillations at high fields and low temperatures. ${ }^{12}$ Zero-field experiments on these well-ordered samples show no evidence of static broken translational symmetry in nuclear-magnetic-resonance $\left(\mathrm{YBa}_{2} \mathrm{Cu}_{4} \mathrm{O}_{8}\right)$ (Ref. 13) or neutron-scattering $\left(\mathrm{YBa}_{2} \mathrm{Cu}_{3} \mathrm{O}_{6.5}\right.$-ortho-II) experiments. ${ }^{14}$ This lead us to conclude that the translational symmetry breaking observed by STM in the BSCCO and $\mathrm{Ca}_{2-x} \mathrm{Na}_{x} \mathrm{CuO}_{2} \mathrm{Cl}_{2}$ samples results from the strong disorder associated with random doping a short-range spin liquid in these cuprates.

In this paper we propose that the checkerboard pattern observed by STM is caused by the local disorder in the $\mathrm{CuO}_{2}$ planes. Our explanation goes back to the observation by Chen, Rice, and Zhang (CRZ) (Ref. 15) that when the STM tip is centered above the planar $\mathrm{O}$ sites, it couples to two tunneling paths through apical $\mathrm{Cl}$ ions above the neighboring $\mathrm{Cu}$ sites and as a consequence interference between these paths plays an important role. This interference leads to a different tunneling matrix elements for electrons into hole states which are bonding (antibonding) upon reflection about the central $\mathrm{O}$ site of the $\mathrm{Cu}-\mathrm{O}-\mathrm{Cu}$ bond. ${ }^{15} \mathrm{CRZ}$ considered the dilute limit and examined the localized states of a single hole bound to a $\mathrm{Na}^{+}$acceptor in a $\mathrm{Ca}_{2-x} \mathrm{Na}_{x} \mathrm{CuO}_{2} \mathrm{Cl}_{2}$ sample. The $\mathrm{Na}^{+}$acceptor sits above the center of a $(\mathrm{CuO})_{4}$ square plaquette. They found a doubly degenerate acceptor bound 


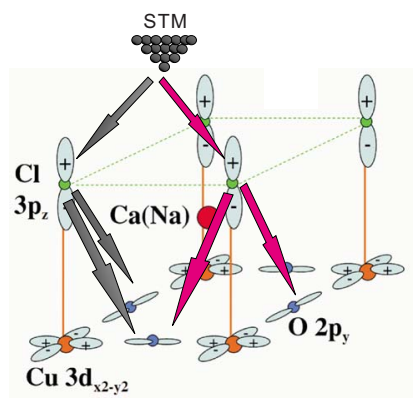

(a)

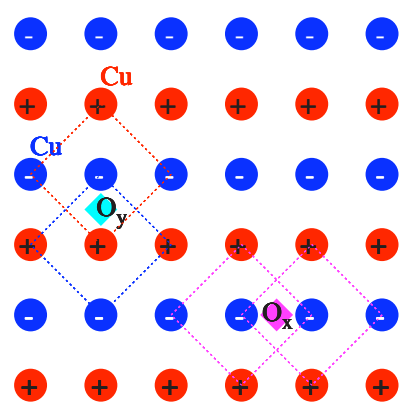

(b)

FIG. 1. (Color online) Top panel: schematic demonstration of the interference of the possible two tunneling paths from STM tip to the $\mathrm{Cu}-\mathrm{O}-\mathrm{Cu}$ hybrid state when the tip is at the midway between two $\mathrm{Cl}$ atoms. Lower panel: the contribution of the antinodal $\boldsymbol{k}_{A, y}$ $=(0, \pi)$ state to the two interference paths. The blue and red dots are $\mathrm{Cu}$ sites with the alternative sign along $\boldsymbol{y}$ direction denoting the sign of the Bloch wave function of antinodal $\boldsymbol{k}_{A, y}$ quasiparticles as shown in Eq. (8). (The signs for the $d_{x^{2}-y^{2}}$ symmetry of $\mathrm{Cu}$ orbitals are not shown.) For the $\boldsymbol{k}_{A, y / x}$ quasiparticle contributions to $\mathrm{O}_{y / x}$ of the two paths cancel each other, meanwhile the contributions to $\mathrm{O}_{x / y}$ of the two paths add together.

state which in the presence of a local quadrupole electric field splits into two states, each with only $C_{2}$ bonding symmetry. However this explanation of the local symmetry reduction from $C_{4}$ to $C_{2}$ symmetry is not compatible with the recent STM data, which shows that the checkerboard pattern is formed by the tunneling of electrons and holes into pseudogap states located in the antinodal regions of $\boldsymbol{k}$ space. The lowest energy for a single hole is at the nodal regions of $\boldsymbol{k}$ space so that single hole acceptor bound states are made up from combinations of hole states near to the nodal not antinodal regions of $\boldsymbol{k}$ space. Nonetheless their main conclusion, that interference of tunneling paths occurs when the STM tip is above an $\mathrm{O}$ site, remains valid.

In light of this new STM data ${ }^{10}$ we concentrate our attention on the role of the antinodal regions of $\boldsymbol{k}$ space as origins of the checkerboard pattern. There are two such regions, one near $(\pi, 0)$ and the other near $(0, \pi)$. The bondingantibonding pattern on the $\mathrm{Cu}-\mathrm{O}-\mathrm{Cu}$ bonds differs between the two antinodal regions. For quasiparticle near the $(0, \pi)$ antinodal region the Bloch phase factor is bonding on $\mathrm{Cu}$ $\mathrm{O}-\mathrm{Cu}$ in the $\boldsymbol{x}$ direction and antibonding in the $\boldsymbol{y}$ direction, as shown in Fig. 1(b). This pattern is reversed for the case of the $(\pi, 0)$ antinodal region. Thus when the STM tip lies above the planar $\mathrm{O}$ sites in a $(\mathrm{CuO})_{4}$ square plaquette the stronger signal comes from the antinodal region with a bonding phase factor and a local difference in the tunneling DOS between the two antinodal regions will cause a breaking of the $C_{4}$ to $C_{2}$ symmetry. This symmetry breaking occurs only when the STM tip lies above the $\mathrm{O}$ sites in a $(\mathrm{CuO})_{4}$ square plaquette but not when the tip is above $\mathrm{Cu}$ sites. Typical checkerboard patterns display strong modulation above the planar $\mathrm{O}$ sites but much weaker modulation is observed at the $\mathrm{Cu}$ sites. This strongly suggests that we should concentrate on possible mechanisms that locally differentiate the two antinodal regions since it is this difference which will show up at the planar $\mathrm{O}$ sites.
In their recent paper, Kohsaka et al. discussed the conditions that enhance the checkerboard pattern in the tunneling maps. They find that the pattern emerges most clearly in $Z$ maps which plot the ratio of differential conductances $g(\boldsymbol{r}, V)$, at opposite bias, i.e.,

$$
Z(\boldsymbol{r}, E=e V)=g(\boldsymbol{r},+V) / g(\boldsymbol{r},-V) .
$$

Further the checkerboard pattern is most pronounced when the voltage $V$ is adjusted locally to the local pseudogap value. This value varies strongly across the field of view in the STM measurements. This is typically $40 a \times 40 a$ and the typical length scale of the variation in the local value of the pseudogap is roughly $4 a$, similar to the length scale of coherent checkerboard patterns.

In this paper we examine possible sources of local symmetry breaking from $C_{4}$ to $C_{2}$ symmetry in the STM patterns in the presence of strong local disorder, combining the CRZ theory for STM tunneling with the Yang, Rice, and Zhang (YRZ) (Ref. 16) phenomenological form for the single electron propagator in the underdoped pseudogap phase.

\section{YRZ-PHENOMENOLOGICAL SINGLE PARTICLE PROPAGATOR}

YRZ based their propagator on a generalization of the form of the propagator for a lightly doped array of two-leg Hubbard ladders derived by Konik et al. ${ }^{17}$ They introduced a self-energy, $\Sigma_{R}(\boldsymbol{k}, \omega, x)$, which diverges at $\omega=0$ on a surface spanned by elastic particle-particle umklapp scattering analogous to the behavior of the ladder model. In the twodimensional square lattice this umklapp surface is a diamond connecting antinodal points on the Brillouin-zone boundary. Note this umklapp surface appears as the energy-gap surface also in the case of wider Hubbard ladders with more than two legs. ${ }^{18}$ YRZ took over the form of $d$-wave RVB gap function $\Delta_{R}(\boldsymbol{k}, x)=\Delta_{0}(x)\left(\cos k_{x}-\cos k_{y}\right)$ from the renormalized mean-field theory (RMFT) of Zhang et al. ${ }^{19}$ Using their Gutzwiller renormalization factor $g_{t}(x)$ for longer range hopping, YRZ proposed a form for the coherent part of the single-particle propagator $G^{P}(\boldsymbol{k}, \omega, x)$ in the pseudogap state,

$$
\begin{gathered}
G^{P}(\boldsymbol{k}, \omega, x)=\frac{g_{t}(x)}{\omega-\boldsymbol{\epsilon}(\boldsymbol{k})-\mu-\Sigma_{R}(\boldsymbol{k}, \omega, x)}=g_{t}(x) \sum_{i} \frac{Z_{\boldsymbol{k}}^{i}}{\omega-E_{\boldsymbol{k}}^{i}}, \\
\Sigma_{R}(\boldsymbol{k}, \omega, x)=\frac{\Delta_{R}^{2}(\boldsymbol{k}, x)}{\omega+\boldsymbol{\epsilon}^{0}(\boldsymbol{k})},
\end{gathered}
$$

where $\epsilon^{0}(\boldsymbol{k})$ only includes the nearest-neighbor (NN) renormalized hopping contribution, meanwhile $\boldsymbol{\epsilon}(\boldsymbol{k})$ includes both the NN and longer range renormalized hopping contributions.

The YRZ propagator fits well the quasiparticle dispersion $E_{k}^{i}$ and their weight $Z_{k}^{i}$ seen in angle-resolved photoemission (ARPES) experiments on the pseudogap phase. ${ }^{16,20}$ It has also been used successfully to interpret a number of other experiments on the cuprate superconductor, i.e., Raman scattering, ${ }^{21}$ optical properties, ${ }^{22}$ and specific heat. ${ }^{23} \mathrm{Re}$ cently Yang et $a l .{ }^{20}$ showed that it also accounts well for the particle-hole asymmetry in the quasiparticle properties open- 

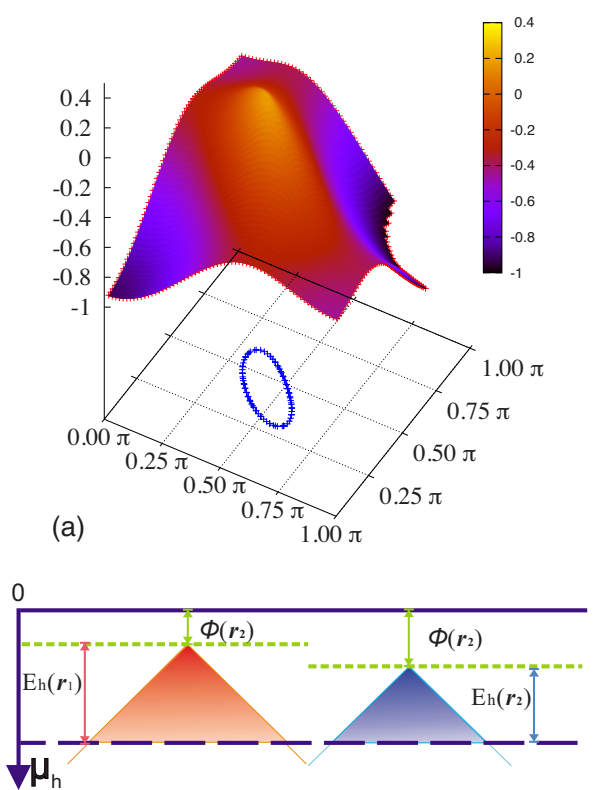

(b)

FIG. 2. (Color online) Top Panel: the low band from the singleparticle propagator Eq. (2) in pseudogap state with the Fermi surface (hole pocket) projected on the $x y$ plane. The bottom of the hole Fermi sea is located at the midway between the node and $(\pi / 2, \pi / 2)$. Lower panel: schematic demonstration of the spatial variation in the hole Fermi energy $E_{h}(\boldsymbol{r})$ due to the random electric field $\phi(r)$.

ing up along the Fermi arcs reported by Yang et al. in the normal pseudogap phase. ${ }^{24}$ Very recently ${ }^{25}$ the temperature and doping dependence of superconducting penetration depth was also shown to fit well to this scheme. In the antinodal regions of $\boldsymbol{k}$ space the energy gap is largest with a magnitude $\Delta_{R}\left(\boldsymbol{k}_{A}, x\right)$, where $\boldsymbol{k}_{A}$ is an antinodal wave vector: $\boldsymbol{k}_{A, x}=(\pi, 0)$ and $\boldsymbol{k}_{A, y}=(0, \pi)$. The value of $\Delta_{0}(x)$ is taken from the renormalized mean-field theory of Zhang et al. ${ }^{19}$ for the RVB state. It is a strong function of the hole density, $x$, dropping linearly with increasing $x$ from $\Delta_{0}$ at $x=0$ to 0 at a critical hole concentration $x=x_{c}(\sim 0.2)$.

\section{RANDOM ELECTRIC FIELD IN A THOMAS-FERMI APPROXIMATION}

In the cuprates $\mathrm{BSCCO}$ and $\mathrm{Ca}_{2-x} \mathrm{Na}_{x} \mathrm{CuO}_{2} \mathrm{Cl}_{2}$ which have been studied in the STM experiments, the holes are introduced by randomly distributed acceptors situated above and below the $\mathrm{CuO}_{2}$ planes. The random electric field $\phi(\boldsymbol{r})$ associated with the acceptors generates a random component in the local hole density. The case of a slowly varying potential can be treated in a Thomas-Fermi approximation. The hole chemical potential $\mu_{h}$ is a constant in space. In the YRZ propagator the hole pocket is determined by the quasiparticle energy contour at the chemical potential $\mu_{h}$ which lies above the minimum hole energy $E_{h}^{b}$ located at the midway between the node and $(\pi / 2, \pi / 2)$ points in hole notation as shown in the upper panel of Fig. 2. The hole DOS rises almost linearly with increasing hole energy in the YRZ ansatz, in agreement with recent angle-integrated photoemission data. ${ }^{26}$ The spa- tial varying hole Fermi energy $E_{h}(\boldsymbol{r})$ relative to the bottom of the hole band in a simple Thomas-Fermi ansatz is given by

$$
E_{h}(\boldsymbol{r})=\mu_{h}-\phi(\boldsymbol{r})
$$

leading to a spatial variation in the hole density $x(\boldsymbol{r})$, as shown in the lower panel of Fig. 2. This has two effects, first, a breathing of the area enclosed by the hole pockets and therefore the Fermi arcs observed by ARPES and by quasiparticle interference in STM. Second, it introduces a local variation in the RVB gap $\Delta_{R}\left(\boldsymbol{k}_{A}, x\right)$ which as remarked above, is a strong function of the hole density. The strong spatial variation in the RVB gap can be measured by the energy of the maximum in the DOS relative to the constant chemical potential. That has been reported in several STM studies. ${ }^{2,10,27}$ A breathing of the Fermi arc determined by quasiparticle interference in STM spectra was recently reported by Wise et al. ${ }^{27}$

\section{INTERFERENCE IN TUNNELING SPECTRA WITH THE STM TIP ABOVE O SITES}

The detailed STM maps showing the checkerboard patterns were taken on two compounds, Dy-doped BSCCO and $\mathrm{Ca}_{2-x} \mathrm{Na}_{x} \mathrm{CuO}_{2} \mathrm{Cl}_{2}$. In BSCCO samples the outmost surface layer is a $\mathrm{BiO}$ plane with the $\mathrm{Bi}$ ions located directly above the $\mathrm{Cu}$ sites in the $\mathrm{CuO}_{2}$ plane. Assuming the STM tips couple predominantly to the empty $6 s / 6 p$ Bi orbitals, the analysis presented below will apply also to BSCCO. We shall restrict ourselves to the former which has a simpler structure. At this point it is useful to recap the CRZ analysis, which discussed the role interference between neighboring tunneling paths in the STM maps. Meanwhile, we focus on the antinodal quasiparticles. With the STM tip centered at position $\boldsymbol{r}$, the differential conductivity at $T=0$ and voltage $V$, is following Tersoff and Hamann, ${ }^{28}$

$$
\frac{d I(\boldsymbol{r})}{d V} \propto \sum_{\sigma, m}\left|\left\langle m\left|a_{\boldsymbol{r}, \sigma}^{\dagger}\right| \Psi_{0}\right\rangle\right|^{2} \delta\left(\omega-E_{m}+E_{0}\right),
$$

where $a_{\boldsymbol{r}, \sigma}^{\dagger}$ is the electron creation operator at position $\boldsymbol{r},|m\rangle$ are final eigenstates with energy $E_{m}$, and $\omega=e V,\left|\Psi_{0}\right\rangle$ is the ground state with energy $E_{0}$. The outermost surface layer is composed of $\mathrm{Cl}$ ions which sit directly above the $\mathrm{Cu}$ ions in the topmost $\mathrm{CuO}_{2}$ layer so that the STM tip couples primarily to their $3 p_{z}$ states (see Fig. 1). When the STM tip is scanned from above the $\mathrm{Cl}$ ion at $i$ to a $\mathrm{NN} \mathrm{Cl-ion} \mathrm{site,} i$ $+\boldsymbol{\tau}[\boldsymbol{\tau}=( \pm 1,0)$ or $(0, \pm 1)]$, we have

$$
\begin{gathered}
a_{\boldsymbol{r}, \sigma}^{\dagger}=\langle\boldsymbol{i}, \mathrm{Cl} \mid \boldsymbol{r}\rangle p_{\mathrm{Cl}, \boldsymbol{i}, \sigma}^{\dagger}+\langle\boldsymbol{i}+\boldsymbol{\tau}, \mathrm{Cl} \mid \boldsymbol{r}\rangle p_{\mathrm{Cl}, \boldsymbol{i}+\boldsymbol{\tau}, \sigma}^{\dagger}, \\
p_{\mathrm{Cl}, \boldsymbol{i}, \sigma}^{\dagger}=\sum_{\boldsymbol{\tau}^{\prime}}\left\langle\boldsymbol{i}+\boldsymbol{\tau}^{\prime}, \mathrm{Cu} \mid \boldsymbol{i}, \mathrm{Cl}\right\rangle c_{\boldsymbol{i}+\boldsymbol{\tau}^{\prime}, \sigma}^{\dagger} \sim \frac{1}{2} \sum_{\boldsymbol{\tau}^{\prime}}(-1)^{\tau_{x}^{\prime}} c_{\boldsymbol{i}+\boldsymbol{\tau}^{\prime}, \sigma}^{\dagger},
\end{gathered}
$$

where $(-1)^{\tau_{x}^{\prime}}$ is from the $d_{x^{2}-y^{2}}$ symmetry of the $\mathrm{Cu}$ orbital, $p_{\mathrm{Cl}, i, \sigma}^{\dagger}$ is the electron creation operator at site $i \mathrm{Cl}$, and $c_{i, \sigma}^{\dagger}$ is the electron creation operator for the $d$ - $p$ hybridized orbital centered on the $\mathrm{Cu}$ site at $\boldsymbol{i}$. The integrated current up to a positive voltage $V$ (electron injection) is then 


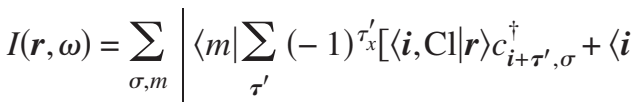

$$
\begin{aligned}
& \left.+\boldsymbol{\tau}, \mathrm{Cl}|\boldsymbol{r}\rangle c_{i+\boldsymbol{\tau}^{\prime}+\boldsymbol{\tau}}^{\dagger}\right]\left.\left|\Psi_{0}\right\rangle\right|^{2} \Theta\left(\omega-E_{m}+E_{0}\right),
\end{aligned}
$$

where $\Theta$ is the Heaviside step function. CRZ pointed out that, when the tip is positioned above the $\mathrm{O}$ ion at $i+\tau / 2$ lying halfway between the two $\mathrm{Cu}$ ions at $i$ and $i+\tau$, the integrated current will be sensitive to the relative phase of the hole states centered at the NN Cu ions. Note the orthogonality of the $\mathrm{Cl} 3 p_{z}$ states to the $d_{x^{2}-y^{2}}$ symmetry of the hole states centered on the $\mathrm{Cu}$ site underneath leads to a dominant hybridization with the hole states centered on the four NN $\mathrm{Cu}$ sites. ${ }^{29}$ Finally, we arrive at the result for the tunneling currents when the STM tip lies halfway between $\mathrm{NN} \mathrm{Cl}$ sites, i.e., above the $\mathrm{O}$ sites in the $\boldsymbol{x} / \boldsymbol{y}$ oriented $\mathrm{Cu}-\mathrm{O}-\mathrm{Cu}$ bonds $I^{\mathrm{O}_{x / y}}(\boldsymbol{r}+\boldsymbol{\tau} / 2, \omega)$ and $I^{\mathrm{Cu}}(\boldsymbol{r}, \omega)$ when the STM tip lies above a $\mathrm{Cu}$ site

$$
\begin{aligned}
I^{\mathrm{O}_{x / y}}= & \Theta\left(\omega-E_{m}+E_{0}\right) \mid\langle m| \sum_{\boldsymbol{\tau}^{\prime}, \sigma}(-1)^{\tau_{x}^{\prime}}\left(c_{\boldsymbol{i}+\boldsymbol{\tau}^{\prime}, \sigma}^{\dagger}+c_{\boldsymbol{i}+\boldsymbol{\tau}+\boldsymbol{\tau}^{\prime}}^{\dagger}\right) \\
& \times\left.\left|\Psi_{0}\right\rangle\right|^{2}, \\
I^{\mathrm{Cu}}= & \Theta\left(\omega-E_{m}+E_{0}\right)\left|\left\langle m\left|\sum_{\boldsymbol{\tau}^{\prime}, \sigma}(-1)^{\tau_{x}^{\prime}} c_{\boldsymbol{i}+\boldsymbol{\tau}^{\prime}, \sigma}^{\dagger}\right| \Psi_{0}\right\rangle\right|^{2} .
\end{aligned}
$$

CRZ considered the case of a single hole bound to a $\mathrm{Na}^{+}$ acceptor which they showed may well have a doubly degenerate ground state. This degeneracy in turn splits in the presence of a quadrupole electric field into states with reflection symmetries with respect to the $x$ and $y$ axes $P_{x}= \pm 1$ and $P_{y}= \pm 1$, i.e., bonding and antibonding states at the $\mathrm{O}$ sites. They showed that this lower $C_{2}$ symmetry shows up in the tunneling current.

As we remarked earlier, the new STM data on underdoped $\mathrm{Ca}_{2-x} \mathrm{Na}_{x} \mathrm{CuO}_{2} \mathrm{Cl}_{2}$ samples show that the checkerboard pattern is associated into antinodal states rather than the single hole bound states considered by CRZ. Note, however, quasiparticles in the antinodal regions states are composed of Bloch states near $\boldsymbol{k}_{A, x}=(\pi, 0)$ and $\boldsymbol{k}_{A, y}=(0, \pi)$, and these have similar reflection properties with opposite parities for reflections about the $\boldsymbol{x}$ and $\boldsymbol{y}$ axes. Focusing on the contributions from antinodal quasiparticles, we can set

$$
c_{\boldsymbol{i}, \sigma}^{\dagger} \sim c_{\boldsymbol{k}_{A, x}}^{\dagger}(-1)^{i_{x}}+c_{\boldsymbol{k}_{A, y}^{\dagger}}^{\dagger}(-1)^{i_{y}}
$$

with the sign alternating pattern from $\boldsymbol{k}_{A, y}$ shown in Fig. 1(b). So that when the tip is above the $\mathrm{O}_{x / y}$ site at $\boldsymbol{i}+\boldsymbol{\tau} / 2$,

$$
\begin{aligned}
a_{i+\tau / 2, \sigma}^{\dagger} & \sim \frac{1}{2} \sum_{\boldsymbol{\tau}^{\prime}}(-1)^{\tau_{x}^{\prime}}\left[c_{\boldsymbol{i}+\boldsymbol{\tau}^{\prime}, \sigma}^{\dagger}+c_{\boldsymbol{i}+\boldsymbol{\tau}^{\prime}+\boldsymbol{\tau}, \sigma}^{\dagger}\right] \\
& \sim \sum_{\boldsymbol{k}_{A, x / y}, \boldsymbol{\tau}^{\prime}}(-1)^{\tau_{x}^{\prime}} c_{\boldsymbol{k}_{A, x / y}^{\dagger}}^{\dagger}(-1)^{\left(i+\boldsymbol{\tau}^{\prime}\right)_{x / y}\left[1+(-1)^{\tau_{x / y}}\right] .}
\end{aligned}
$$

From the factor $1+(-1)^{\tau_{x / y}}$, it is obvious that for $\mathrm{O}_{x / y}$ the contribution from the $\boldsymbol{k}_{A, x / y}$ quasiparticles vanishes, mean-

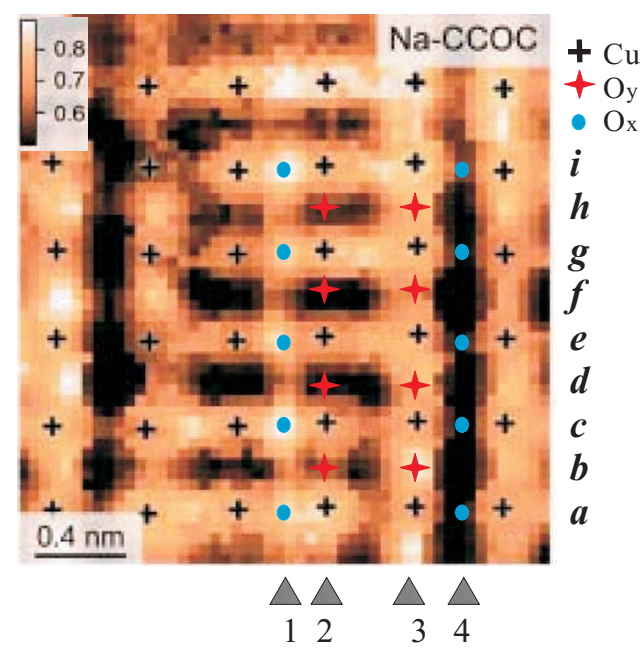

FIG. 3. (Color online) Typical checkerboard pattern revealed by $\mathrm{R}$ map in Na-CCOC observed in one recent STM experiments Ref. 2 (reproduced from original paper). The blue solid circles (red solid stars) show the position of the oxygen atoms on $\mathrm{Cu}-\mathrm{O}-\mathrm{Cu}$ bond along $\boldsymbol{x} / \boldsymbol{y}$ directions [labeled by the combination of vertical lines (1-4) and horizon (a-i)].

while that from $\boldsymbol{k}_{A, y / x}$ is enhanced due to the interference effect, as schematically shown in Fig. 1. As a result any local perturbation which breaks the symmetry between the two antinodal regions of $\boldsymbol{k}$ space will lead to a lower $C_{2}$ symmetry in the tunneling current. This leads us then to look at various sources that can give rise to such local symmetry breaking in this highly random system. To proceed further it is useful to examine the nature of the anisotropy observed in the tunneling spectra more closely.

\section{MEASURED ANISOTROPIC STM TUNNELING SPECTRA}

Kohsaka et al. ${ }^{10}$ found that the checkerboard pattern emerges most clearly in $\mathrm{Z}$ maps which plot the ratio of differential conductances $g(\boldsymbol{r}, V)$, at opposite bias, when the voltage $V$ is chosen to be at the local value of the antinodal energy gap. In an earlier publication by the same group, individual spectra $g(\boldsymbol{r}, V)$, where plotted for a series of tip positions placed above $\mathrm{O}$ sites in a small area with a pronounced checkerboard modulation. ${ }^{2}$ This is illustrated in the checkerboard modulation which shows up clearly in the $\mathrm{R}$ map in Fig. 3 which is reproduced from their Fig. 4(b) ${ }^{2}$ The $\mathrm{R}$ ratio measures the ratio of the integrated currents $I(\boldsymbol{r}, V)$, at positive and negative voltages

$$
R(\boldsymbol{r}, E=e V)=I(\boldsymbol{r},+V) / I(\boldsymbol{r},-V) .
$$

The map reproduced in Fig. 3 shows the $\mathrm{R}$ pattern taken at an energy $E=150 \mathrm{meV}$, with clear modulations when the tip 

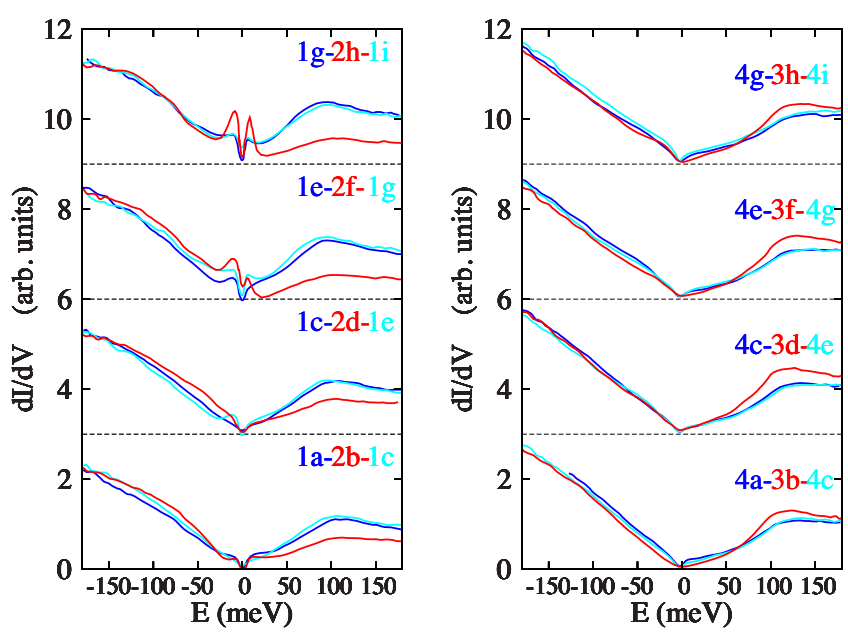

FIG. 4. (Color online) Differential tunneling conductance spectra taken on oxygen sites along the vertical lines $(1,2,3,4)$ as indicated in Fig. 3, reproduced from Kohsaka et al. (Ref. 10). Each subpanel shows the comparison of $d I / d V$ between the two neighboring $\mathrm{O}_{x} \mathrm{~s}$ (blue and cyan) and one $\mathrm{O}_{y}$ (red) on each $(\mathrm{Cu}-\mathrm{O}-\mathrm{Cu})_{4}$ palette (with the position shown in Fig. 3). Note that the spectra is normalized to have the same intensity at negative voltages to facilitate the comparison and demonstrate the lowering of the symmetry from $C_{4}$ to $C_{2}$.

is above the $\mathrm{O}$ sites with a local $C_{2}$ symmetry but almost no modulations are observed over the $\mathrm{Cu}$ sites (denoted by black crosses). The value of the $\mathrm{R}$ ratio over the $\mathrm{O}$ sites ranges from 0.9 (shown as white) to 0.5 (black).

The individual differential conductances taken above a series of $\mathrm{O}$ and $\mathrm{Cu}$ sites were also shown in their Fig. 5(b). We have extracted the individual tunneling spectra $g(\boldsymbol{r}, V)$ above the $\mathrm{O}$ sites in this typical small area with a pronounced checkerboard pattern in the R map [shown in Fig. 5b of Ref. 2]. All spectra on these underdoped samples show a substantial asymmetry between negative and positive voltages. ${ }^{30,31}$ The negative voltage spectra, which correspond to electron extraction or hole injection, show little structure and has the larger differential conductances. Since a hole can easily exchange positions with a neighboring occupied site while an electron can only hop onto the unoccupied neighboring sites, the tunneling processes that accompany an injected hole are much stronger than those for an electron, as shown by Anderson and $\mathrm{Ong}^{30}$ and by Randeria et al. ${ }^{31}$ Within the renormalized mean-field theory of Zhang et al., ${ }^{19}$ the asymmetry is largely due to the incoherent tunneling process in the hole injection. In the approach we use here based on the YRZ normal-state propagator, only coherent parts of the tunneling processes are included and the particle-hole asymmetry appears at finite voltages which involves tunneling into quasiparticle states away from the chemical potential. The positive voltage spectra show a clear peak around an energy of order $100 \mathrm{meV}$. Such a peak appears in the density of states calculated for the YRZ propagator by Yang et al. ${ }^{16}$ The YRZ propagator is an ansatz to describe coherent quasiparticles moving in a RVB background and so should agree better for positive voltage due to the weaker contribution of inelastic processes which accompany electron injection. In the subsequent discussion we will focus on the positive volt-
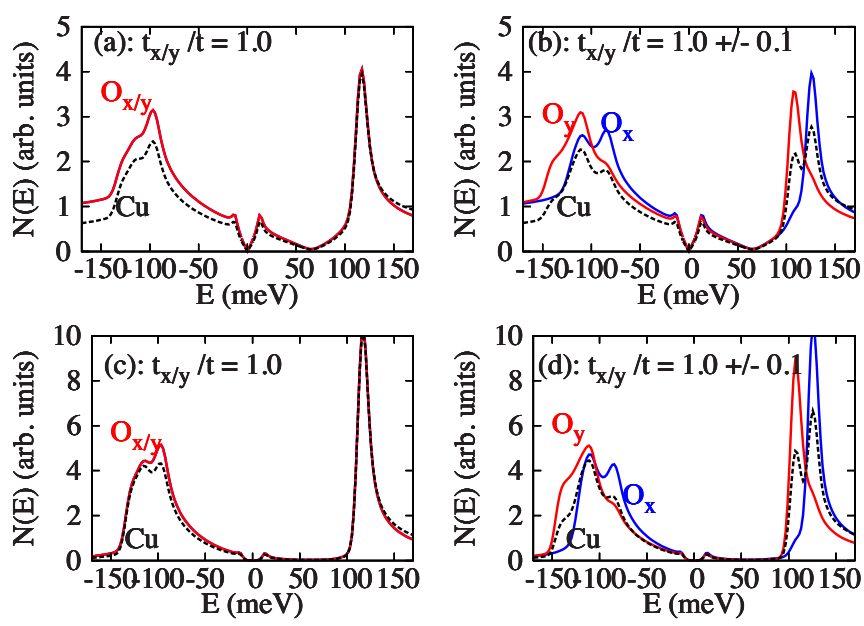

FIG. 5. (Color online) The density of states on $\mathrm{Cu}$ and two kinds oxygen sites $\left[\mathrm{O}_{x / y}\right]$ from YRZ propagator Eq. (11) without (panels a and $\mathrm{b}$ ) and with (panels $\mathrm{c}$ and $\mathrm{d})$ the interference factor $\left(\cos k_{x}\right.$ $\left.-\cos k_{y}\right)^{2}$. Panels (b and d) are the results in the presence of an intrinsic $C_{2}$ asymmetry in the NN hopping integrals along $\boldsymbol{x}$ and $\boldsymbol{y}$ directions $t_{x / y} / t=1 \pm 0.1$. The splitting of the peaks at positive voltage shows up clearly. The other parameters are as following: $\Delta_{0}$ $=0.4, t^{\prime}=-0.3, t^{\prime \prime}=0.2$, doping $x=0.10$ (all energies are of unit $t$ $=300 \mathrm{meV}$ ). Very small superconducting gaps are added to each band in pseudogap state, which does not change the RVB gap behavior (the two pronounced peaks at high energy $\sim 100 \mathrm{meV}$ ) on $\mathrm{O}_{x / y}$ (Ref. 2).

age spectra and normalize the spectra to be the same in the negative voltage region. In Fig. 4 we show a series of the differential conductances with the tip above $\mathrm{O}$ sites in the $\boldsymbol{x} / \boldsymbol{y}$ oriented $\mathrm{Cu}-\mathrm{O}-\mathrm{Cu}$ bonds. The difference from the lower $C_{2}$ symmetry is clearly visible.

\section{POSSIBLE SOURCES OF THE ANISOTROPY IN THE TUNNELING SPECTRA}

We now discuss a number of physical effects which could cause an anisotropy in the tunneling spectra.

\section{A. Intrinsic $C_{2}$ asymmetry due to nematic or Pomeranchuk order}

An asymmetry between the two antinodal regions near $\boldsymbol{k}_{A, x}=(\pi, 0)$ and $\boldsymbol{k}_{A, y}=(0, \pi)$ suggests that an intrinsic instability which breaks the $C_{4}$ symmetry may be present in these samples. The possibility of such an instability has been discussed extensively in the literature. One set of these proposals focuses on a quantum electronic liquid crystal with nematic order. ${ }^{32-35}$ A second on a Pomeranchuk instability which splits the two Van Hove singularities. ${ }^{36,37}$ Recent neutronscattering measurements of the low-frequency spin fluctuations in YBCO samples have been interpreted as evidence for such intrinsic instabilities. ${ }^{38}$ In the presence of intrinsic asymmetry the NN hopping matrix elements in the $\boldsymbol{x}$ and $\boldsymbol{y}$ directions $t_{x}$ and $t_{y}$ will be slightly different. Assuming that the intrinsic asymmetry in the self-energy in the YRZ propagator is small, the change in $\mathrm{NN}$ hopping integrals $t_{x / y}$ leads to a relative displacement of the reference energy for the 
RVB splitting at $\boldsymbol{k}_{A, x}$ and $\boldsymbol{k}_{A, y}$. This in turn leads to a shift in the position of the energy gaps $\Delta_{R}\left(\boldsymbol{k}_{A}, x\right)$, relative to the constant chemical potential. This is apparent in Fig. 5 which shows the predicted anisotropy arising from a uniform hopping asymmetry.

The density of states, $N(E)$, observed by putting the STM tip above $\mathrm{O}_{x / y}$ and $\mathrm{Cu}$ sites takes the following form from Eq. (7):

$$
\begin{gathered}
N^{\mathrm{O}_{x / y}}(E) \sim \sum_{i, \boldsymbol{k}} Z_{\boldsymbol{k}}^{i} \cos ^{2} \frac{k_{x / y}}{2}\left(\cos k_{x}-\cos k_{y}\right)^{2} \delta\left(E_{\boldsymbol{k}}^{i}-E\right), \\
N^{\mathrm{Cu}}(E) \sim \sum_{i, \boldsymbol{k}} Z_{\boldsymbol{k}}^{i}\left(\cos k_{x}-\cos k_{y}\right)^{2} \delta\left(E_{\boldsymbol{k}}^{i}-E\right),
\end{gathered}
$$

where $\left(\cos k_{x}-\cos k_{y}\right)^{2}$ is the interference factor due to the hybridization with the hole states centered on the four NN $\mathrm{Cu}$ sites as discussed earlier.

In a clean well-ordered sample with intrinsic $C_{2}$ asymmetry order large domains should occur but in the highly disordered $\mathrm{Ca}_{2-x} \mathrm{Na}_{x} \mathrm{CuO}_{2} \mathrm{Cl}_{2}$ samples there is a strong spatially varying electric field. The linear coupling between the orientation of the domains and the local quadrupole component of a random electric field acts as a random orienting field on the order. Since the symmetry of the order is Ising type, the random orienting field will act similarly to a random field in an Ising model. It is well known that the ground state of this Ising model consists of a random array of domains, consistent with the orientational domains observed in the checkerboard patterns. A word of caution, however, is in order. The local anisotropy in the STM spectra does not seem to be consistent with that predicted for this type local asymmetry order. The spectra in Fig. 4 show a $C_{2}$-modulation pattern arising from an overall reduction in the positive voltage spectra, rather than a shift in the maxima that would signal the presence of this intrinsic $C_{2}$ asymmetry in Fig. 5 .

The theoretical DOS for the STM tip located above $\mathrm{Cu}$ and $\mathrm{O}$ sites shown in Fig. 5 display considerably sharper structure in both cases, with and without the weighting factor $\left(\cos k_{x}-\cos k_{y}\right)^{2}$, than is evident in the experimental conductances in Fig. 4. The theoretical DOS contain two prominent features. One is the approximately linear drop from negative to positive voltages leading to the minimum at $\simeq 60 \mathrm{meV}$. Interestingly this linear feature has been reported in integrated photoemission studies on BSSCO samples by Hashimoto et al. ${ }^{26}$ as discussed recently by Yang et al. ${ }^{20}$ In the STM spectra there are signs of a minimum at positive voltages in the spectral $2 \mathrm{~h}$ and $2 \mathrm{f}$ in Fig. 4 but at a smaller voltage and faint signs in the other spectra. The second feature is the very sharp peak at $\sim 100 \mathrm{meV}$ in the theoretical spectra and the much broader peaks at around the same energy in the STM spectra. For both discrepancies a possible remedy could be the much stronger disorder broadening in the local probe in the STM spectra as compared to spatially averaged spectra observed in photoemission.

\section{B. Random electric fields}

The random distribution of $\mathrm{Na}^{+}$acceptors in $\mathrm{Ca}_{2-x} \mathrm{Na}_{x} \mathrm{CuO}_{2} \mathrm{Cl}_{2}$ samples generates a random electric field. The local quadrupole component of this field acting on a
$(\mathrm{CuO})_{4}$ plaquette will couple differently to the states at $\boldsymbol{k}_{A, x}$ and $\boldsymbol{k}_{A, y}$. The former is antibonding on the $\mathrm{Cu}-\mathrm{O}-\mathrm{Cu}$ bonds along the $\boldsymbol{x}$ axis and bonding on the $\mathrm{Cu}-\mathrm{O}-\mathrm{Cu}$ bonds along the $\boldsymbol{y}$ axis while the latter has the opposite bonding pattern. These patterns cause opposite quadrupole charge distributions in the two states leading to a potential splitting of the antinodal states in the presence of an external electric quadrupole field. This potential splitting will generate STM spectra similar to those we found for hopping asymmetry and so it also differs from the anisotropy displayed in Fig. 4

\section{Variations in the quasiparticle weight and in the localization length}

The tunneling DOS into quasiparticle states is weighted by the quasiparticle weight in the single-particle propagator. In the YRZ ansatz this weight is taken from the RMFT calculations of Zhang et al. ${ }^{19}$ and is simply proportional to the hole density, $x$. However the fact that little change in the energy gaps $\Delta_{R}\left(\boldsymbol{k}_{A, x}, x\right)$ is evident in the spectra shown in Fig. 4 argues against such an explanation. Also the spatial variation in the hole density is longer ranged than the distance between $\mathrm{O}$ sites on a $(\mathrm{CuO})_{4}$. Another possibility is that there is substantial anisotropy in the disorder scattering in $\boldsymbol{k}$ space which causes a local anisotropy in the localization lengths. An anisotropic energy shift and localization length could result, if the potential scattering from the disorder is mainly between opposite antinodal points rather than between $\pm \boldsymbol{k}_{A, x}$ and $\pm \boldsymbol{k}_{A, y}$. An anisotropic energy shift, for example, could remove weight from the energy range (200 $\mathrm{meV}$ ) covered in the STM experiments. The somewhat longer range of the disorder potential is consistent with this suggestion. The umklapp correlated scattering wave vector for backscattering between the antinodal regions near $\pm \boldsymbol{k}_{A, x}$ (or $\pm \boldsymbol{k}_{A, y}$ ) is much smaller than the wave vector connecting the two antinodal regions, $\simeq(\pi, \pi)$. The Fourier transforms of the checkerboard maps show peaks arising from shortrange order in the form of $4 a$ wide unidirectional electronic domains. The analysis presented here does not directly address the presence of short-range order on a length scale $>a$. Several authors have suggested that the wave vector of this short-range order is approximately equal to the wave vector connecting parallel pieces of the bare band-structure Fermi surface near the saddle points ${ }^{11}$ and also the wave vector connecting the turning points of the quasiparticle contours in the normal-state YRZ propagators. ${ }^{39}$ The length scale of the domains at $\sim 4 a$ is roughly the same as the disorder potential length scale but the interpretation of the short-range order in the checkerboard patterns remains to be an open issue.

\section{CONCLUSION}

In this paper we have examined several possible origins for the local checkerboard pattern in the tunneling DOS measured over $\mathrm{O}_{x / y}$ sites. The presence of interference between two tunneling paths centered above the neighboring $\mathrm{Cu}$ sites 
leads to an anisotropic weighting of the two antinodal regions in $\boldsymbol{k}$ space. Thus the local $C_{2}$ pattern of the checkerboard can reflect an asymmetry between the two antinodal $\boldsymbol{k}$-space regions. An asymmetric hopping $\left(t_{x}, t_{y}\right)$ could arise from an intrinsic instability, e.g., toward nematic or Pomeranchuk order. This would split the energies of the pseudogaps at $\pm \boldsymbol{k}_{A, x}$ and $\pm \boldsymbol{k}_{A, y}$. However, an examination of the measured anisotropy in the differential conductance points rather toward an asymmetry in the average magnitude of the conductance, which possibly could result from a difference in the localization lengths at the two antinodal saddle points in the quasiparticle dispersion.

\section{ACKNOWLEDGMENTS}

We are very grateful to J. C. S. Davis and Y. Kohsaka for permission to reproduce their STM data and Y. Chen for stimulating discussions. Support from the MANEP program of the Swiss National funds (K.-Y. Y. and T. M. R.), and RGC grant of HKSAR (W.-Q. Chen and F.C.Z.) is gratefully acknowledged. This research was supported in part by the National Science Foundation under Grant No. PHY0551164.
${ }^{1}$ For a recent review see K. M. Shen and J. C. S. Davis, Mater. Today 11, 14 (2008).

${ }^{2}$ Y. Kohsaka, C. Taylor, K. Fujita, A. Schmidt, C. Lupien, T. Hanaguri, M. Azuma, M. Takano, H. Eisaki, H. Takagi, S. Uchida, and J. C. Davis, Science 315, 1380 (2007).

${ }^{3}$ L. Balents, L. Bartosch, A. Burkov, S. Sachdev, and K. Sengupta, Phys. Rev. B 71, 144509 (2005).

${ }^{4}$ Z. Tesanovic, Nat. Phys. 4, 408 (2008).

${ }^{5}$ M. Vojta and O. Rosch, Phys. Rev. B 77, 094504 (2008).

${ }^{6}$ M. Granath, Phys. Rev. B 77, 165128 (2008).

${ }^{7}$ Eun-Ah Kim, M. J. Lawler, P. Oreto, S. Sachdev, E. Fradkin, and S. A. Kivelson, Phys. Rev. B 77, 184514 (2008).

${ }^{8}$ A. Pelissetto, S. Sachdev, and E. Vicari, Phys. Rev. Lett. 101, 027005 (2008).

${ }^{9}$ M. Vojta, Phys. Rev. B 78, 144508 (2008).

${ }^{10}$ Y. Kohsaka, C. Taylor, P. Wahl, A. Schmidt, Jhinhwan Lee, K. Fujita, J. W. Alldredge, K. McElroy, Jinho Lee, H. Eisaki, S. Uchida, D.-H. Lee, and J. C. Davis, Nature (London) 454, 1072 (2008).

${ }^{11}$ Q. H. Wang and D. H. Lee, Phys. Rev. B 67, 020511(R) (2003).

${ }^{12}$ D. LeBoeuf, N. Doiron-Leyraud, J. Levallois, R. Daou, J.-B. Bonnemaison, N. E. Hussey, L. Balicas, B. J. Ramshaw, Ruixing Liang, D. A. Bonn, W. N. Hardy, S. Adachi, C. Proust, and L. Taillefer, Nature (London) 450, 533 (2007).

${ }^{13}$ I. Tomeno, T. Machi, K. Tai, N. Koshizuka, S. Kambe, A. Hayashi, Y. Ueda, and H. Yasuoka, Phys. Rev. B 49, 15327 (1994).

${ }^{14}$ C. Stock, W. J. L. Buyers, Z. Tun, R. Liang, D. Peets, D. Bonn, W. N. Hardy, and L. Taillefer, Phys. Rev. B 66, 024505 (2002); C. Stock, W. J. L. Buyers, R. Liang, D. Peets, Z. Tun, D. Bonn, W. N. Hardy, and R. J. Birgeneau, ibid. 69, 014502 (2004).

${ }^{15}$ Yan Chen, T. M. Rice, and Fu-Chun Zhang, Phys. Rev. Lett. 97, 237004 (2006).

${ }^{16}$ K.-Y. Yang, T. M. Rice, and Fu-Chun Zhang, Phys. Rev. B 73, 174501 (2006).

${ }^{17}$ R. M. Konik, T. M. Rice, and A. M. Tsvelik, Phys. Rev. Lett. 96, 086407 (2006).

${ }^{18}$ K. L. Hur and T. M. Rice, Ann. Phys. 324, 1452 (2009).

${ }^{19}$ F. C. Zhang, C. Gros, T. M. Rice, and H. Shiba, Supercond. Sci. Technol. 1, 36 (1988).

${ }^{20}$ K.-Y. Yang, H. B. Yang, P. D. Johnson, T. M. Rice, and Fu-Chun
Zhang, EPL 86, 37002 (2009)

${ }^{21}$ B. Valenzuela and E. Bascones, Phys. Rev. Lett. 98, 227002 (2007).

${ }^{22}$ E. Illes, E. J. Nicol, and J. P. Carbotte, Phys. Rev. B 79, 100505(R) (2009).

${ }^{23}$ J. P. F. LeBlanc, E. J. Nicol, and J. P. Carbotte, Phys. Rev. B 80, 060505(R) (2009).

${ }^{24}$ H.-B. Yang, J. D. Rameau, P. D. Johnson, T. Valla, A. Tsvelik, and G. D. Gu, Nature (London) 456, 77 (2008).

${ }^{25}$ J. P. Carbotte, K. A. G. Fisher, J. P. F. LeBlanc, and E. J. Nicol, arXiv:0909.3814 (unpublished).

${ }^{26}$ M. Hashimoto, T. Yoshida, K. Tanaka, A. Fujimori, M. Okusawa, S. Wakimoto, K. Yamada, T. Kakeshita, H. Eisaki, and S. Uchida, Phys. Rev. B 79, 140502(R) (2009).

${ }^{27}$ W. D. Wise, K. Chatterjee, M. C. Boyer, T. Kondo, T. Takeuchi, H. Ikuta, Zhijun Xu, Jinsheng Wen, G. D. Gu, Yayu Wang, and E. W. Hudson Nat. Phys. 5, 213 (2009).

${ }^{28}$ J. Tersoff and D. R. Hamann, Phys. Rev. B 31, 805 (1985).

${ }^{29}$ A. V. Balatsky, I. Vekhter, and J.-X. Zhu, Rev. Mod. Phys. 78, 373 (2006)

${ }^{30}$ P. W. Anderson and N. P. Ong, J. Phys. Chem. Solids 67, 1 (2006).

${ }^{31}$ M. Randeria, R. Sensarma, N. Trivedi, and F. C. Zhang, Phys. Rev. Lett. 95, 137001 (2005).

${ }^{32}$ S. A. Kivelson, E. Fradkin, and V. J. Emery, Nature (London) 393, 550 (1998).

${ }^{33}$ S. A. Kivelson, I. P. Bindloss, E. Fradkin, V. Oganesyan, J. M. Tranquada, A. Kapitulnik, and C. Howald, Rev. Mod. Phys. 75, 1201 (2003).

${ }^{34}$ V. Oganesyan, S. A. Kivelson, and E. Fradkin, Phys. Rev. B 64, 195109 (2001).

${ }^{35}$ Y.-J. Kao and H.-Y. Kee, Phys. Rev. B 72, 024502 (2005).

${ }^{36}$ H. Yamase, V. Oganesyan, and W. Metzner, Phys. Rev. B 72, 035114 (2005).

${ }^{37}$ C. J. Halboth and W. Metzner, Phys. Rev. Lett. 85, 5162 (2000).

${ }^{38}$ V. Hinkov, D. Haug, B. Fauque, P. Bourges, Y. Sidis, A. Ivanov, C. Bernhard, C. T. Lin, and B. Keimer, Science 319, 597 (2008).

${ }^{39}$ E. Bascones and B. Valenzuela, Phys. Rev. B 77, 024527 (2008). 